\title{
Perioperative Management and Outcomes in Children with Hereditary Bleeding Disorder: a Retrospective Review at a Single Hemophilia Treatment Center
}

\author{
Ha Nuel Chon, Ja Hyang Cho and Young Shil Park
}

Department of Pediatrics, Kyung Hee University Hospital at Gangdong, Seoul, Korea

\begin{abstract}
Background: Establishing hemostasis for surgical procedures in children with hereditary bleeding disorders is challenging. We evaluated the results of surgical procedures in children with hereditary bleeding disorders at our center and reviewed the preoperative management and hemorrhagic complications.

Methods: We conducted a retrospective electronic medical record review from October 2006 to September 2019. Children with hereditary bleeding disorders who had elective surgeries or emergency operations were identified by an electronic record search. The primary focus was a review of clotting factor replacement strategies and bleeding complications.
\end{abstract}

Results: In total, 126 elective procedures and 4 emergency surgeries were performed on 95 children at our center. Of the 95 children, hemophilia A, hemophilia B, von Willebrand disease, and factor VII deficiency were 74, 15, 4, and 2, respectively. The main disease distribution of procedures was 99 with hemophilia A, 24 with hemophilia B, and 4 with von Willebrand disease. Procedures included various orthopedic surgeries $(87 / 130,66.9 \%)$, placement or revision of a central venous catheter $(8 / 130$, $6.2 \%)$, and otolaryngology procedures (7/130, 5.4\%). All patients received preoperative clotting factor replacement followed by various postoperative clotting factor replacement regimens. Thirteen procedures (10.0\%) in five children were performed in the presence of high titers of clotting factor inhibitors. No deaths or life-threatening bleeding occurred after any procedure. Nine of the 130 procedures $(6.9 \%)$ were complicated by postoperative bleeding. Tonsillectomy and adenoidectomy were the most common procedures complicated by hemorrhage (3/5, 60\%).

Conclusion: Surgical procedures are safe in children with hereditary bleeding disorders with adequate preparation and replacement of clotting factors. Bleeding remains a problem in a subset of patients and requires ongoing hematological involvement and oversight. Delayed bleeding following tonsillectomy was particularly common and suggests a need for close follow-up and ongoing factor administration for this group of patients.

Key Words: Bleeding disorder, Hemophilia, von Willebrand disease, Surgical procedure, Perioperative
pISSN 2233-5250 / elSSN 2233-4580 https://doi.org/10.15264/cpho.2020.27.2.113 Clin Pediatr Hematol Oncol 2020;27:113 119

Received on August 18, 2020

Revised on October 5, 2020

Accepted on October 19, 2020
Corresponding Author: Young Shil Park Department of Pediatrics, Kyung Hee University Hospital at Gangdong, 892 Dongnam-ro, Gangdong-gu, Seoul 05278, Korea Tel: +82-2-440-6133 Fax: +82-2-440-7175

E-mail: pysmd@khnmc.or.kr ORCID ID: orcid.org/0000-0002-6643-4245

\section{Introduction}

Undertaking surgical procedures in children with he- reditary bleeding disorders is demanding due to the management of hemostasis [1]. Surgeries on children with a hereditary bleeding disorder are often hesitating for fear of bleeding complications. The management of children 
with a hereditary bleeding disorder has evolved and various factor concentrates are available. Prophylactic clotting factor concentrates have been used widely in children with severe hemophilia [2,3]. Numerous techniques and protocols are available for factor replacement therapy before surgical procedures in children with a hereditary bleeding disorder [4]. Treatment decision depends on the patient's status and it is typically individualized. However, the goal of each treatment approach is to prevent bleeding that may occur in intraoperative and postoperative conditions.

Various methods of management preoperative/postoperative bleeding on patients with hereditary bleeding disorder are already known [4]. However, there are not many reports of the progress or outcome of surgery in children with a hereditary bleeding disorder. Therefore, in this study, we evaluated the results of surgical procedures in children with a hereditary bleeding disorder at our center and reviewed the preoperative management and hemorrhagic complications.

\section{Materials and Methods}

The electronic medical records from surgeries and procedures of children with a hereditary bleeding disorder were reviewed from October 2006 to September 2019 at Kyung Hee University Hospital at Gangdong, Seoul, Korea.

Previously known patients and newly diagnosed patients during the preoperative evaluation period were included. Demographic data were collected, including age, gender, type of hereditary bleeding disorder, and types of surgical procedures and complications.

The preoperative evaluation at our center includes a complete blood cell count, prothrombin time (PT), and activated partial thromboplastin time (aPTT) [5,6]. Baseline clotting factor level and antibodies to clotting factors were also determined in patients with a known hereditary bleeding disorder. The severity of hemophilia is classified on the baseline clotting factor level of the patient; severe $(<1 \%)$, moderate $(1-5 \%)$, or mild $(>5 \%)$ [7].

Treatment-related data, including the type of clotting factor concentrates, and postoperative complications in- cluding re-hospitalization, bleeding, and transfusion were collected.

In patients with hereditary bleeding disorder, clotting factor can be administered by continuous infusion or bolus infusion. A standardized protocol of bolus infusion was used under the supervision of a multidisciplinary hemophilia team at our institute. The first bolus of $50 \mathrm{IU} / \mathrm{kg}$ for hemophilia A and $80 \mathrm{IU} / \mathrm{kg} \times 1.3-1.4$ for hemophilia $B$ was injected 15-30 min before the surgical procedure for patients without clotting factor inhibitors. The target preoperative factor level was $80-100 \%$ regardless of the severity of hemophilia. Approximately $50 \%$ of the initial dose was injected every 8-12 hours. Recombinant factor VIIa was administered intravenously at doses of $15-30 \mu \mathrm{g} /$ kg every $4 \mathrm{~h}$ postoperatively during the first $24-48 \mathrm{~h}$ for a factor VII deficiency. Changes in doses depended on the patient's postoperative status, including factor levels, bleeding, and whether they underwent major surgery. The dose interval was increased to 24 hours after postoperative days 1-7. Target factor levels were tailored based on the patients' status 3 days after the first major surgery.

In this study, continuous infusion was only considered for emergency surgeries. In the case of continuous infusion, a standardized protocol was used. The first bolus continuous infusion dose was $50 \mathrm{IU} / \mathrm{kg}$ and administered 30 min before the incision on the day of surgery. The infusion rate started at $4 \mathrm{IU} / \mathrm{kg} / \mathrm{h}$ and was controlled to the status of the patient and clinical situation by measuring factor VIII levels daily. After the first 3 days of operation, the infusion rate was altered to get target factor VIII levels of $80 \%$.

\section{Results}

In total, 126 elective procedures and 4 emergency surgeries were performed on 95 children at our center. The median age of the patients was 12.0 years (range, $0-18$ years). Of 95 children, 92 were male and 3 were female and hemophilia A, hemophilia B, von Willebrand disease (VWD), and factor VII deficiency were 74, 15, 4, and 2, respectively. In the surgical procedures, the distribution of hereditary bleeding disorders was as follows: hemo- 
philia A, 99; hemophilia B, 24; VWD, 4; and Factor VII deficiency, 3. Severe type hemophilia A included 77 cases, moderate type included 8 , and the mild type came to 14. Severe type hemophilia B included 17 and the moderate type came to 7 cases (Table 1). Multiple surgical procedures were reported in some patients.

Four patients were newly diagnosed using the routine preoperative laboratory data. There were two cases of mild hemophilia A, one VWD, and one factor VII deficiency (Table 2). All of these newly diagnosed patients underwent surgeries well with appropriate administration of clotting factors.

The procedures included synovectomy (64/130, 49.2\%), other orthopedic procedures $(23 / 130,17.7 \%)$, central venous catheter placement or revision $(8 / 130,6.2 \%)$, surgical extraction of a molar $(1 / 130,0.8 \%)$, and tonsillectomy and adenoidectomy (5/130, 3.8\%) (Table 3).

All patients received preoperative factor replacement followed by various postoperative factor replacement

Table 1. Distribution of hereditary bleeding disorders

\begin{tabular}{lrr}
\hline Hereditary bleeding disorders & N & $N^{*}$ \\
\hline Hemophilia A & 74 & 99 \\
Severe & 55 & 77 \\
Moderate & 8 & 8 \\
Mild & 11 & 14 \\
Inhibitor (+) & 3 & 5 \\
Hemophilia B & 15 & 24 \\
Severe & 9 & 17 \\
Moderate & 6 & 7 \\
Mild & 0 & 0 \\
Inhibitor (+) & 2 & 8 \\
Von Willebrand disease & 4 & 4 \\
Factor VII deficiency & 2 & 3 \\
Total & 95 & 130 \\
\hline
\end{tabular}

*Number of surgical procedures. regimens. Thirteen procedures (10.0\%) in 5 children were performed in the presence of high titers of clotting factor inhibitors without any complications. Three patients received activated prothrombin complex concentrates, 5

Table 3. Types of surgeries and procedures

\begin{tabular}{lc}
\hline \multicolumn{1}{c}{ Surgeries and procedures } & N \\
\hline Orthopedic surgery & 64 \\
Synovectomy & 22 \\
Non synovectomy & 1 \\
Miscellaneous (skin graft) & 8 \\
Central venous line procedures & \\
ENT surgical procedures & 5 \\
Tonsillectomy and adenoidectomy & 1 \\
Myringotomy & 1 \\
Lingual frenotomy & 1 \\
Dental (molar extraction) & \\
General surgery & 4 \\
Laparoscopic appendectomy & 1 \\
Appendectomy & 1 \\
Laparotomy & 1 \\
Perianal abscess incision & 3 \\
Hernia & 1 \\
Gastric bypass with gastrojejunostomy* & \\
Anal fistulotomy & 2 \\
Neurosurgery & 1 \\
Craniotomy* & 1 \\
Thoracolumbar spinal fusion & \\
Cervical spinal fusion* & 1 \\
Ophthalmologic surgery & 1 \\
Therapeutic evacuation of anterior chamber & 1 \\
Repair of blepharoptosis & 1 \\
Extropia operation & 1 \\
Thoracic and cardiovascular surgery & 1 \\
Circumcision & \\
Hydrocele excision & 1 \\
Orchiopexy & \\
Retrograde intrarenal surgery & 1 \\
\hline
\end{tabular}

*Emergency operation.

Table 2. Newly diagnosed cases

\begin{tabular}{llcll}
\hline Patient & \multicolumn{1}{c}{ Surgery } & Preoperative evaluation & Diagnosis & \multicolumn{1}{c}{ Complications } \\
\hline $16 \mathrm{y} / \mathrm{F}$ & Non synovectomy & prolonged aPTT & VWD & None \\
$7 \mathrm{y} / \mathrm{M}$ & Non synovectomy & prolonged aPTT & HA (mild, Ab-) & Rebleeding after T \& A \\
$10 \mathrm{y} / \mathrm{M}$ & T \& A & prolonged aPTT & HA (mild, Ab-) & None \\
$6 \mathrm{y} / \mathrm{F}$ & T \& A & prolonged aPTT & FVII deficiency & None \\
\hline
\end{tabular}

aPTT, activated partial thromboplastin time; VWD, von Willebrand disease; HA, hemophilia A; Ab, clotting factor inhibitors; $\mathrm{T}$ \& $\mathrm{A}$, tonsillectomy and adenoidectomy; FVII, Factor VII. 
patients were controlled using recombinant activated Factor VII, and 5 patients used sequential bypassing therapy.

The median duration from surgery to discharge was 9.1 days (range, 0-42 days). No deaths or life-threatening bleeding occurred after any procedure. Four emergency operations required intensive care unit care (Table 4, Fig. 1 ). Nine of the 130 procedures (6.9\%) were complicated by postoperative bleeding, and transfusions were needed in patients during 5 of the 130 procedures (3.8\%). Tonsillectomy and adenoidectomy was the most common procedure complicated by hemorrhage $(3 / 5,60 \%)$. In these cases, no bleeding problems were seen immediately after tonsillectomy and adenoidectomy, but the patients were readmitted for delayed bleeding (Table 5).

Table 4. Cases of emergency operation

\begin{tabular}{|c|c|c|c|c|c|c|c|}
\hline Patient & Diagnosis & Surgery & ICU care & HD & Transfusion & Factor infusion & Prognosis \\
\hline $\begin{array}{l}8 \mathrm{~m} / \mathrm{M} \\
\mathrm{HA} \text { (Severe, } \\
\mathrm{Ab}-\text { ) }\end{array}$ & $\begin{array}{l}\text { Traumatic ICH Rt. } \\
\text { Sized } 3.9 \times 4.5 \times \\
8.4 \mathrm{~cm}\end{array}$ & $\begin{array}{l}\text { Craniotomy and } \\
\text { hematoma } \\
\text { evacuation }\end{array}$ & Yes & 37 & No & $\begin{array}{l}\text { Factor VIII } \\
\text { concentrates } \\
\mathrm{Cl} \& \mathrm{BI}\end{array}$ & $\begin{array}{l}\text { Convulsions at the time of } \\
\text { hospitalization, using } \\
\text { anticonvulsants, convulsions } \\
\text { were controlled well. }\end{array}$ \\
\hline $\begin{array}{l}14 \mathrm{y} / \mathrm{M} \\
\mathrm{HA} \text { (Severe, } \\
\mathrm{Ab}-\text { ) }\end{array}$ & $\begin{array}{l}\text { Epidural hematoma } \\
\text { (C3-5) }\end{array}$ & $\begin{array}{l}\text { Cervical posterior } \\
\text { decompression }\end{array}$ & Yes & 40 & Yes & $\begin{array}{l}\text { Factor VIII } \\
\text { concentrates } \\
\mathrm{Cl} \& \mathrm{BI}\end{array}$ & Quadriplegia remained. \\
\hline $\begin{array}{l}15 \mathrm{y} / \mathrm{M} \\
\mathrm{HA} \text { (Severe, } \\
\mathrm{Ab}+\text { ) }\end{array}$ & Hemothorax Rt. & $\begin{array}{l}\text { 8th ICS minimal } \\
\text { lateral } \\
\text { thoracotomy }\end{array}$ & Yes & 40 & Yes & $\begin{array}{l}\text { APCC } \\
\mathrm{BI}\end{array}$ & None \\
\hline $\begin{array}{l}12 \mathrm{y} / \mathrm{M} \\
\mathrm{HA} \text { (Severe, } \\
\mathrm{Ab}-\text { ) }\end{array}$ & $\begin{array}{c}\text { Huge hematoma at } \\
\text { duodenal serosa }\end{array}$ & Gastrojejunostomy & Yes & 11 & Yes & $\begin{array}{l}\text { Factor VIII } \\
\text { concentrates } \\
\mathrm{Cl} \& \mathrm{BI}\end{array}$ & None \\
\hline
\end{tabular}

$I C U$, intensive care unit; $H D$, duration of hospitalization; $H A$, hemophilia $A$; $A b$, clotting factor inhibitors; $I C H$, intracranial hemorrhage; $\mathrm{Cl}$, continuous infusion; $\mathrm{BI}$, bolus infusion; ICS, intercostal space; APCC, activated prothrombin complex concentrates.
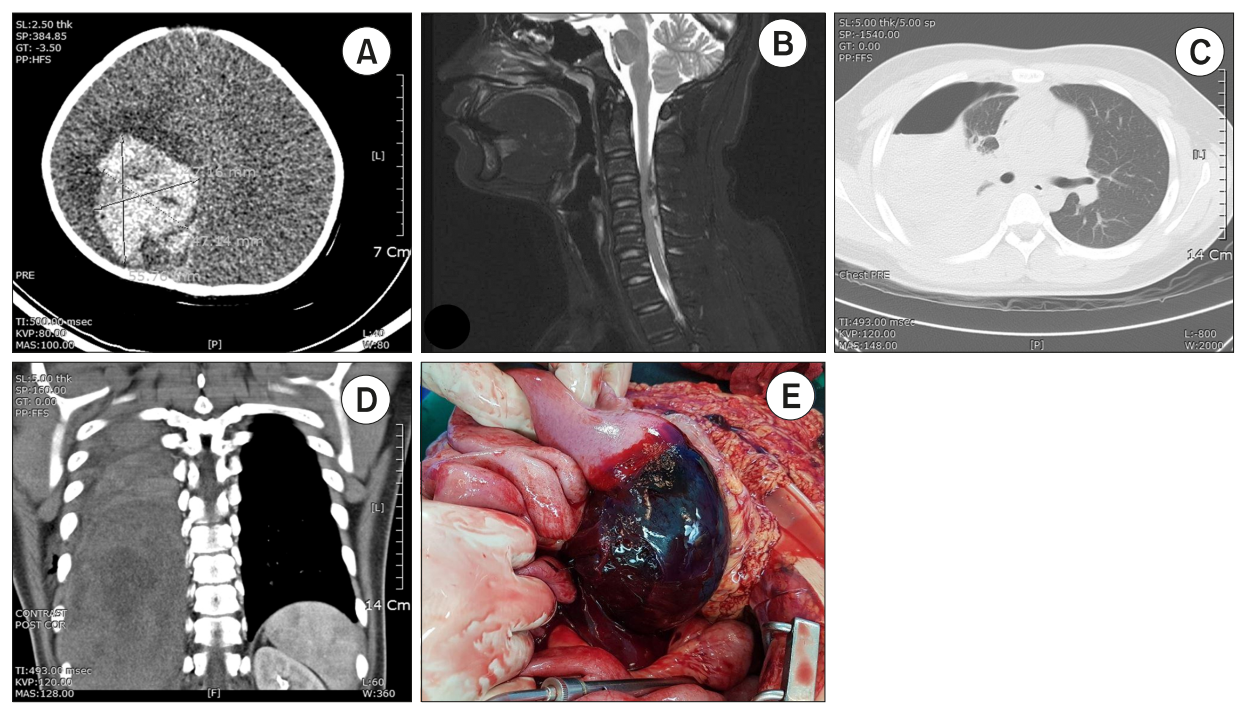

Fig. 1. Cases that underwent emergency surgery. (A) Brain computed tomography (CT) scan of an 8-month-old boy with hemophilia $A$ and traumatic intracranial hemorrhage on the right. A $3.9 \times 4.5 \times 8.4 \mathrm{~cm}$-sized intraventricular hemorrhage showing midline shift to the left side. (B) Magnetic resonance imaging scan of a 14-year-old boy with hemophilia A. Image showing spinal epidural hematoma at level C3-5 along the cervicothoracic spine. (C) and (D) Chest CT scan of a 15-year-old boy with hemophilia A and a traumatic right hemothorax showing a thickened visceral pleural on the right lung. (E) The photograph was taken during explorative laparotomy of a 12-year-old boy with hemophilia A. Unremovable huge hematoma formed inside the duodenum serosa underneath Trietz's ligament. 
Table 5. Tonsillectomy and adenoidectomy cases

\begin{tabular}{|c|c|c|c|c|c|}
\hline Patient & Diagnosis & $\mathrm{HD}$ & Rebleeding date & Anti-fibrinolytic agent & Rebleeding symptom and finding \\
\hline $6 \mathrm{y} / \mathrm{F}$ & FVII deficiency & 6 & & TXA & \\
\hline \multirow[t]{2}{*}{$5 \mathrm{y} / \mathrm{M}$} & HA(Moderate, $\mathrm{Ab}-$ ) & 8 & & & Bloody saliva \\
\hline & & $4^{*}$ & POD 7 & & $\begin{array}{l}\text { Found blood clot on tonsil } \\
\text { but no active bleeding }\end{array}$ \\
\hline \multirow[t]{3}{*}{$6 \mathrm{y} / \mathrm{M}$} & HA (Severe, $\mathrm{Ab}-$ ) & 4 & & \multirow{3}{*}{ TXA } & Bloody vomitus \\
\hline & & $3^{*}$ & POD 10 & & Found blood clot on tonsil \\
\hline & & $8^{*}$ & POD 18 & & but no active bleeding \\
\hline $4 \mathrm{y} / \mathrm{M}$ & HB (Moderate, $\mathrm{Ab}-$ ) & 5 & & & \\
\hline \multirow[t]{2}{*}{$10 \mathrm{y} / \mathrm{F}$} & $\mathrm{HA}($ Mild, $\mathrm{Ab}-$ ) & 3 & & TXA & Bloody vomitus \\
\hline & & $4^{*}$ & POD 11 & TXA & $\begin{array}{l}\text { Found blood clot on tonsil } \\
\text { but no active bleeding }\end{array}$ \\
\hline
\end{tabular}

No transfusion was done.

HD, duration of hospitalization; FVII, factor VII; TXA, tranexamic acid; HA, hemophilia A; Ab, clotting factor inhibitors; POD, postoperative day; HB, Hemophilia B.

*Duration of hospitalization when the patients readmitted.

\section{Discussion}

Hereditary bleeding disorders are a group of complex and heterogeneous diseases. VWD and hemophilia are the most common of these diseases at $1 \%$ and 1 in every 5,000 male births, respectively, and the frequency of a factor VII deficiency is 1 in 300,000 to 1 in 500,000 [810]. Children require surgery for various situations, i.e., congenital problems, hemophilia-related complications, and bleeding due to traumatic events.

Various perioperative regimens for managing bleeding have been described [4], but there have been few reports on the outcomes or prognoses of surgery in children with hereditary bleeding disorders. Therefore, this study aimed to determine the progress of various surgeries performed that have been conducted at a single center in patients with various hereditary bleeding disorders for 13 years.

All 130 cases were administered clotting factors before the surgery or procedure. None of the patients died. Four cases required intensive care, and emergency surgery was performed in all four cases. Two of the four cases were related to trauma. Acute bleeding events are often associated with trauma in children with hereditary bleeding disorders and may progress to surgery if necessary. Clotting factors were administered by continuous in- fusion during emergency surgery for acute bleeding because more aggressive administration of coagulation factors is required in these cases $(3 / 4)$. The use of continuous infusion clotting factors may be a good way to maintain constant clotting factor concentrations during major surgery or with the patient in an emergent status, which reduces the risk of bleeding caused by low factor trough levels $[11,12]$. Some articles reported the continuous infusion method might reduce the total factor VIII dose required and also the incidence of severe bleeding events compared with a bolus infusion. Although there has been little experience in children, there were no adverse side effects of continuous infusion itself in this study.

Among the 95 patients who underwent surgery, four had not been previously diagnosed with any bleeding disorder but were newly diagnosed during a preoperative examination. All had a mild type of hereditary bleeding disorder, but clotting factor concentrates were administered during the surgery. No postoperative bleeding was detected and none of the patients required administration of clotting factors after surgery.

The most common surgical procedure was orthopedic surgery due to hemophilia and related complications. Hemophilic arthropathy is caused by repeated joint bleeding and can be prevented by regular administration of 
coagulation factors [13]. Many Korean children with hemophilia are regularly administered clotting factors, but some patients still need to be operated on with complications, such as those with hemophilic arthropathy. Joint bleeding repeated in the same joint at a young age eventually progresses to joint arthropathy, which affects exercise and school activities in children [14,15]. An arthroscopic synovectomy may help to prevent recurrent hemarthrosis [16].

As mentioned in some reports, tonsillectomy and adenoidectomy is commonly performed in children and has been reported to result in frequent bleeding in children with a hereditary bleeding disorder. The frequency of delayed bleeding cases other than hemorrhagic disease is $1.5-7 \%$ in the general population [17]. No bleeding problems were observed immediately after surgery in the five cases, but they were hospitalized again for rebleeding after discharge. Rebleeding occurred on a postoperative day between 7 and 11. Although the patients readmitted due to the delayed bleeding, when confirmed by a laryngoscope, all had no active bleeding. During the hospitalization period, the patient's diet gradually changed from soft food to an ordinary meal and was discharged after confirming no bleeding.

As many years of experience were accumulated at our center, collaboration with the surgical department was well established, and even if complications occurred or emergency operation needed, we were able to cope well. For successful perioperative management of patients with bleeding disorder, careful planning and experienced multidisciplinary team would be important.

In conclusion, surgical procedures are safe in children with a hereditary bleeding disorder after adequate planning and replacement of clotting factors [6,18]. Bleeding remains a problem in a subset of patients and requires ongoing hematological involvement and supervision. Delayed bleeding following tonsillectomy and adenoidectomy was particularly common and suggests a need for close follow-up and ongoing factor coverage for this group of patients.

\section{Conflict of Interest Statement}

The authors have no conflict of interest to declare.

\section{References}

1. Watts RG, Cook RP. Operative management and outcomes in children with congenital bleeding disorders: a retrospective review at a single haemophilia treatment centre. Haemophilia 2012;18:421-5.

2. Berntorp E, Boulyjenkov V, Brettler D, et al. Modern treatment of haemophilia. Bull World Health Organ 1995;73:691701.

3. Coppola A, Di Capua M, Di Minno MN, et al. Treatment of hemophilia: a review of current advances and ongoing issues. J Blood Med 2010;1:183-95.

4. Hermans C, Altisent C, Batorova A, et al. Replacement therapy for invasive procedures in patients with haemophilia: literature review, European survey and recommendations. Haemophilia 2009;15:639-58

5. Chee YL, Crawford JC, Watson HG, Greaves M. Guidelines on the assessment of bleeding risk prior to surgery or invasive procedures. British Committee for Standards in Haematology. Br J Haematol 2008;140:496-504.

6. Greaves M, Watson HG. Approach to the diagnosis and management of mild bleeding disorders. J Thromb Haemost 2007; 5 Suppl 1:167-74.

7. Kliegman RM, Geme JW St. Nelson textbook of pediatrics. 21st ed. Philadelphia: Elsevier, 2019;2595.

8. Centers for Disease Control and Prevention. Von Willebrand Disease. Washington, DC: U.S. Department of Health \& Human Services, 2019. (Accessed May 25, 2020, at https://www.cdc.gov/ ncbddd/vwd/facts.html)

9. Centers for Disease Control and Prevention. Hemophilia. Washington, DC: U.S. Department of Health \& Human Services, 2020. (Accessed May 25, 2020, at https://www.cdc.gov/ncbddd/ hemophilia/facts.html)

10. National Institutes of Health. Factor VII deficiency. Bethesda, MD: U.S. National Library of Medicine, 2016. (Accessed May 25, 2020, at https://ghr.nlm.nih.gov/condition/factor-vii-deficiency\#statistics)

11. Batorova A, Martinowitz U. Intermittent injections vs. continuous infusion of factor VIII in haemophilia patients undergoing major surgery. Br J Haematol 2000;110:715-20.

12. Meijer K, Rauchensteiner S, Santagostino E, et al. Continuous infusion of recombinant factor VIII formulated with sucrose in surgery: non-interventional, observational study in patients with severe haemophilia A. Haemophilia 2015;21:e19-25.

13. Journeycake JM, Miller KL, Anderson AM, Buchanan GR, Finnegan M. Arthroscopic synovectomy in children and adolescents with hemophilia. J Pediatr Hematol Oncol 2003;25: 726-31.

14. Dunn AL, Busch MT, Wyly JB, Sullivan KM, Abshire TC. Arthroscopic synovectomy for hemophilic joint disease in a pediatric population. J Pediatr Orthop 2004;24:414-26. 
15. Gamble JG, Bellah J, Rinsky LA, Glader B. Arthropathy of the ankle in hemophilia. J Bone Joint Surg Am 1991;73:1008-15.

16. Kaya Bicer E, Kayaokay K, Alsina A, Gunay H, Kavakli K, Aydoğdu S. Role of arthroscopic debridement of hemophilic ankles. Foot Ankle Int 2018;39:1199-204.

17. García-Matte R, María Constanza Beltrán M, Ximena Fonseca
A, Pamela Zúñiga C. Management of children with inherited mild bleeding disorders undergoing adenotonsillar procedures. Int J Pediatr Otorhinolaryngol 2012;76:291-4.

18. Cooper JD, Smith KJ, Ritchey AK. A cost-effectiveness analysis of coagulation testing prior to tonsillectomy and adenoidectomy in children. Pediatr Blood Cancer 2010;55:1153-9. 